\title{
Citrons acides au goût aigre : la lutte chypriote pour l'indépendance (1955-1959) vue par Lawrence Durrell
}

\section{Maria Zerva et Panagiota Anagnostou}

\section{OpenEdition}

Journals

Édition électronique

URL : http://journals.openedition.org/rbnu/654

DOI : $10.4000 /$ rbnu. 654

ISSN : 2679-6104

Éditeur

Bibliothèque nationale et universitaire de Strasbourg

\section{Édition imprimée}

Date de publication : 1 novembre 2017

Pagination : 24-27

ISBN : 9782859230678

ISSN : 2109-2761

\section{Référence électronique}

Maria Zerva et Panagiota Anagnostou, « Citrons acides au goût aigre : la lutte chypriote pour l'indépendance (1955-1959) vue par Lawrence Durrell », La Revue de la BNU [En ligne], 16 | 2017, mis en ligne le 01 juillet 2019, consulté le 11 décembre 2020. URL : http://journals.openedition.org/rbnu/654 DOI : https://doi.org/10.4000/rbnu.654 


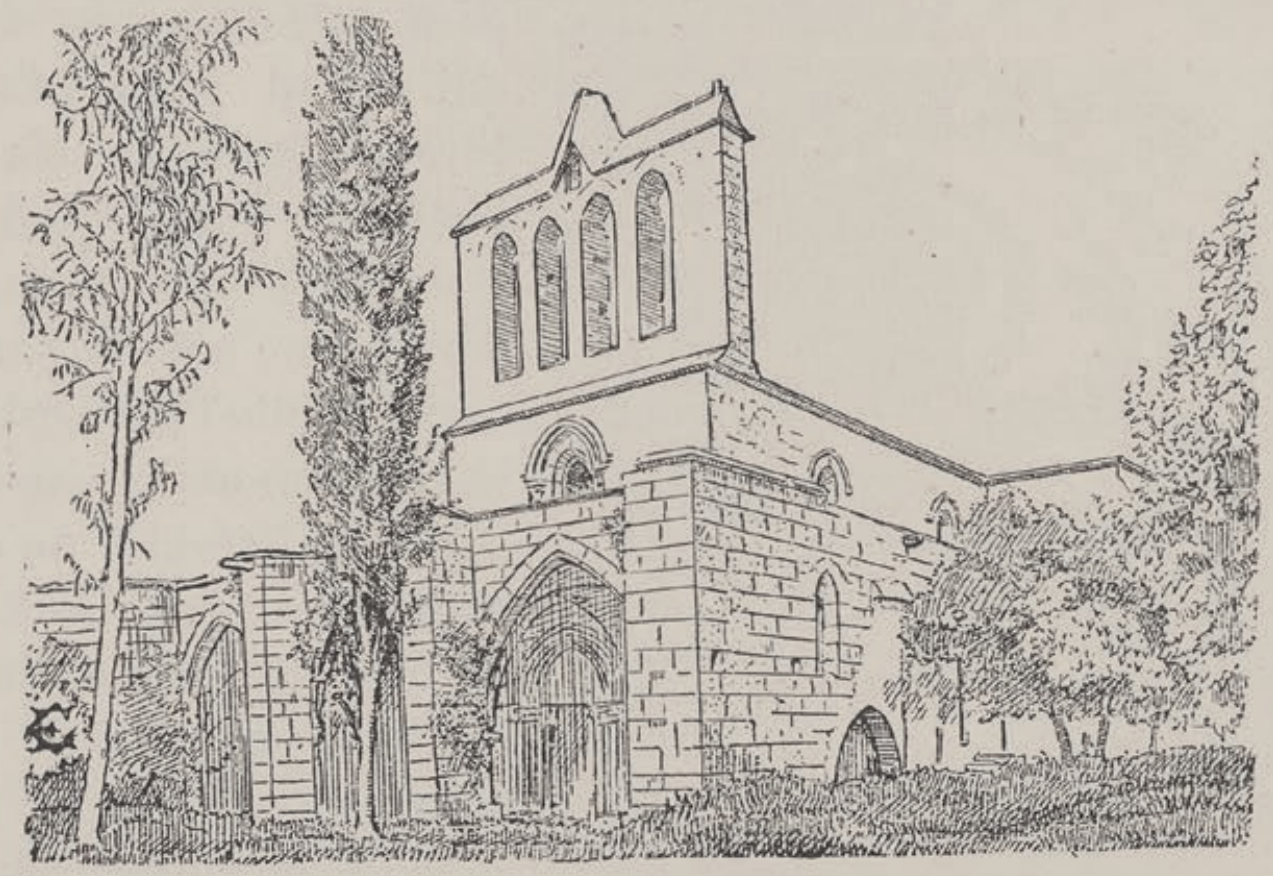

Fig. 149. - Parvis

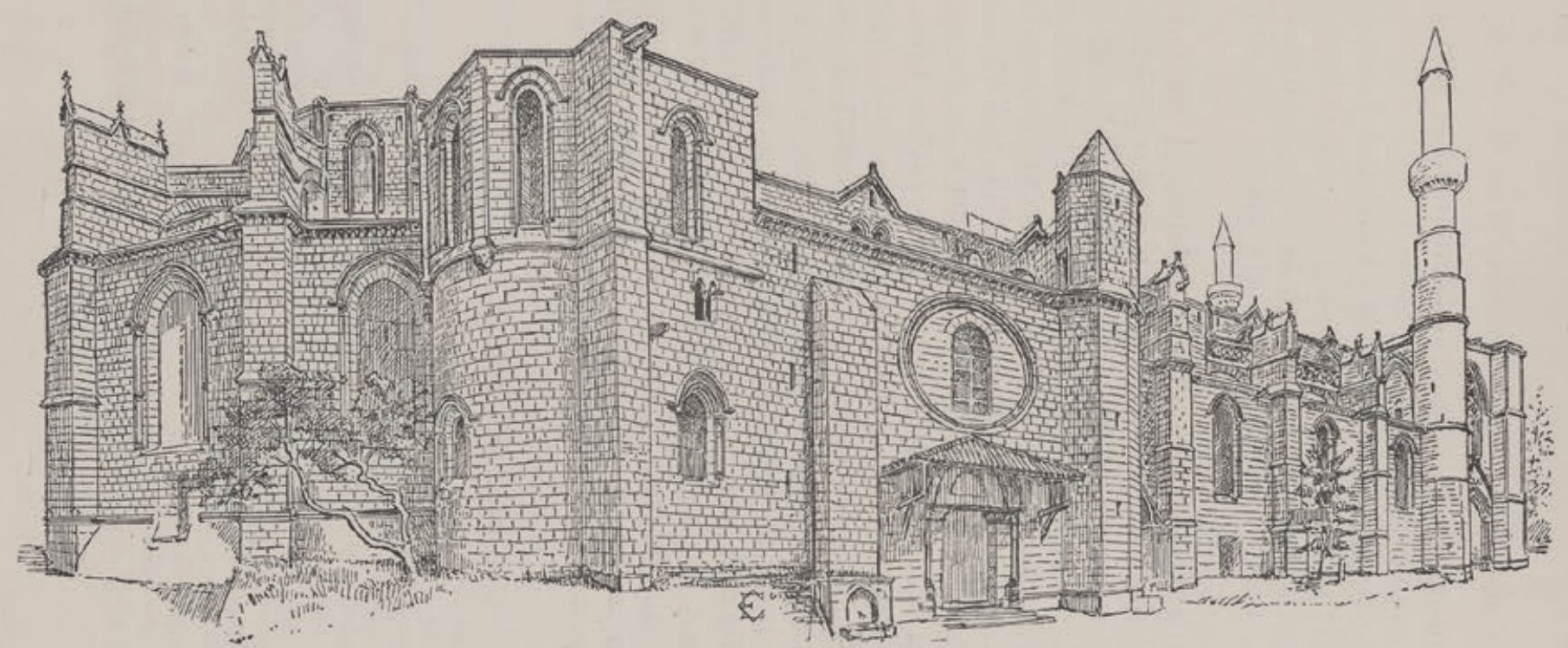

Fig. 28. - Église metropolitaine Sainte-Sophie de Nicosie. 


\section{$\overline{\text { CHYPRE }}$ \\ CITRONS ACIDES AU GOÛT AIGRE : LA LUTTE CHYPRIOTE POUR L'INDÉPENDANCE (1955-1959) VUE PAR LAWRENCE DURRELL \\ PAR MARIA ZERVA ET \\ PANAGIOTA ANAGNOSTOU}

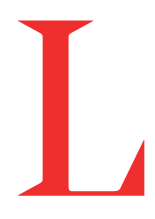

orsque Lawrence Durrell publie son livre Bitter Lemons en 1957, Chypre se trouve toujours prise dans sa lutte pour son indépendance du colon britannique (1955-1959). Le livre est salué par la critique et paraît dans une traduction française en 1961, aux éditions Buchet/Chastel, sous le titre Citrons acides. Bitter Lemons est publié alors que Chypre se trouve encore dans la tourmente, mais que son auteur est dorénavant définitivement installé en France et semble heureux de s'être échappé du chaos chypriote ${ }^{2}$.

Il s'agit du récit des trois ans que Durrell a passés à Chypre, entre 1953 et 1956. Dans la première partie du livre, il fait part au lecteur de son enchantement face à cette île méditerranéenne et de son bonheur de retrouver le monde grec : plus de $80 \%$ de la population insulaire se définit alors comme chypriote grecque. Il connaît ce monde depuis ses séjours à Corfou et à Rhodes, à travers ses lectures, mais aussi les liens qu'il a tissés avec l'intelligentsia grecque, notamment avant l'éclatement de la Seconde Guerre mondiale. C'est d'ailleurs grâce à Durrell qu'Henry Miller fait connaissance de la vie athénienne et des intellectuels appartenant à la génération des années 1930, comme le poète Georges Séféris et le critique littéraire Georges Katsimbalis, à qui est consacré le Colosse de Maroussi de l'écrivain américain (1941).

Durrell dépeint merveilleusement une île à la fois grecque et orientale, empreinte des cultures des « différentes invasions " ${ }^{3}$. De passage à Nicosie, il admire la "grande mosquée aux pointes fragiles, les contours imprécis de ses bastions médiévaux $\|^{4}$ (voir ill. ci-contre, en bas). Il s'agit de la cathédrale gothique Sainte-Sophie, aujourd'hui mosquée Selimiye, construite par les croisés qui sont restés sur l'île de 1191 à 1489 et y ont laissé de nombreuses marques architecturales, avant de céder leur place aux Vénitiens, eux-mêmes évincés par les Ottomans en 1571. Ces vestiges d'art gothique, parsemés dans l'île, sont présentés dans le livre L'art gothique et la Renaissance en Chypre de C. Enlart (1899), d'où proviennent les figures illustrant cet article ${ }^{5}$.

Durrell s'installera finalement au village de Bellapais, surplombé par l'abbaye gothique du même nom, à $8 \mathrm{~km}$ de Kyrenia (voir ill. ci-contre, en haut). 
L'achat et les travaux effectués dans sa nouvelle maison occupent une bonne partie du livre qui se veut « une simple étude à la manière impressionniste de l'état d'esprit et de l'atmosphère de Chypre pendant les années troublées 1953-1956 », et aucunement " un ouvrage politique ", comme il prévient dès les premières lignes de sa préface ${ }^{6}$. De prime abord, il s'agit du récit de voyage aux touches candides d'un prétendu philhellène qui trouve les Chypriotes sympathiques, bien que pas très malins et plutôt rustiques, et regrette la modernité qui vient se substituer à un mode de vie insulaire et archaïque plein de charme.

Il y mène une vie insouciante et goûte à tous les plaisirs que Chypre a à lui offrir : la nature, le vin, l'amitié. Durrell redoute la révolte chypriote car elle mettra une fin à cette insouciance. Lorsqu'il occupera un poste de professeur d'anglais au lycée grec de Nicosie, il se confrontera à la jeunesse de l'île et essaiera de comprendre comment il est possible de combiner un tel amour pour les Anglais avec le désir ardent de l'« Enosis » (union). Ce mot, omniprésent sur l'île, résume les revendications chypriotes grecques : le rattachement à la Grèce. Durrell, flegmatique, prend les précautions nécessaires pour apparaître comme un observateur neutre et impartial de la situation qui s'échauffe. Il critique souvent les Britanniques et l'administration coloniale de l'île, qu'il connaîtra de l'intérieur lorsqu'il occupera le poste d'attaché de presse.

Durrell n'est pas favorable à l'Enosis, mais sa position se durcira avec sa nouvelle fonction. Conscient que cette nomination pourra lui coûter ses amitiés grecques, il n'hésite pas à mettre en avant cet avantage de pénétration dans les cercles grecs afin de «calmer, mais non [d']éradiquer les sentiments favorables à l'Enosis " ${ }^{7}$. Effectivement, ses amitiés grecques en pâtiront et les intellectuels grecs prendront leurs distances avec Durrell, qui n'a plus rien d'un philhellène à leurs yeux. Ainsi son ami d'antan, Séféris, écrit au néohelléniste G. P. Savvidis être très méfiant « en voyant des hommes d'esprit qui sont aussi nos amis (ex. Durrell) servir la propagande de ces Messieurs [les députés britanniques de la Chambre des communes] et utiliser même les amitiés qu'ils avaient encore en Grèce, pour pénétrer et asservir des consciences sur l'île ${ }^{8}$. De même, le peintre chypriote Adamantios Diamantis écrit à Séféris " God save us from the philhellenes ", en pensant à Durrell ${ }^{9}$.
En effet, Durrell adopte le point de vue britannique. Condescendant envers les Chypriotes, il se montre impitoyable envers ceux qui prennent part à la lutte armée pour l'indépendance : ce sont des terroristes, attisés par les "glapissements envenimés " de la propagande venant de la radio d'Athènes, que les villageois chypriotes « [écoutent] sans comprendre, comme des enfants $[\ldots] »^{10}$. Lorsque «le premier assassin terroriste $"{ }^{11}$, le combattant d'EOKA (Organisation nationale des combattants chypriotes) Michalis Karaolis, est arrêté et condamné à mort par pendaison, il lui est impossible de s'y opposer, comme le fait Albert Camus dans son article «L'enfant grec », publié le 6 décembre 1955.

Les Citrons acides véhiculent donc un point de vue colonial, dissimulé derrière leur apparente modération. Le livre est lu encore aujourd'hui comme une description sans parti pris de la situation à Chypre au milieu des années 1950, et son poids dans le marché littéraire est conforté par les critiques positives voire élogieuses. Or son appréciation reste incomplète si l'on ne prend pas en compte les réactions littéraires que les Citrons acides ont suscitées dans le monde grec, réactions qui font partie de l'écriture postcoloniale.

Ainsi, en 1960, l'écrivain grec Rodis Roufos publie à Londres L'Âge de bronze, qui porte comme soustitre Le roman de la lutte chypriote. Il paraîtra en grec la même année mais, de manière significative, quelques semaines après le texte anglais ${ }^{12}$. Roufos écrit son livre directement en anglais, ayant pris, trois ans plus tôt, la mesure de la publication des Citrons acides et jugé de la nécessité de présenter un autre point de vue. Lui-même diplomate, il a passé à Chypre deux années pendant la période critique entre 1954 et 1956, s'est impliqué personnellement dans la lutte chypriote et a participé ensuite aux négociations de Londres qui ont abouti à l'indépendance de $1960{ }^{13}$. Le sous-titre, comportant le mot « roman », insiste sur le fait qu'il s'agit bien d'une fiction, inspirée certes de faits réels, et critique par là aussi les Citrons acides qui font passer la fiction pour la réalité ${ }^{14}$. L'Âge de bronze reste toutefois largement ignoré par le public anglophone, tandis que les pages faisant référence aux Citrons acides sont censurées par l'éditeur anglais Heinemann ${ }^{15}$. L'autre objection littéraire au roman de Durrell viendra de la plume du chypriote Kostas Montis. Nouvelle écrite en grec en 1964 et traduite en anglais seulement en 2004, ses Portes 
closes ${ }^{16}$ peignent également un visage des combattants et de la lutte chypriotes aux antipodes de celui présenté par Durrell : des jeunes inspirés par des valeurs morales et des idéaux supérieurs luttent pour la liberté de leur patrie et subissent une répression impitoyable. Or ces deux réponses restent encore aujourd'hui largement méconnues du public non hellénophone, tandis que les Citrons acides dominent le marché littéraire mondial et véhiculent une représentation littéraire partiale de la lutte chypriote pour l'indépendance des années 19551959.

\section{NOTES}

1- Raisins aigres (Sour grapes) est le terme utilisé par l'écrivain grec Rodis Roufos pour se référer aux Citrons acides dans L'Âge de bronze (voir ci-dessous). Ce nom ne laissait aucun doute pour un public avisé, en raison d'un compte rendu des Citrons acides publié en 1957 dans les Times of Cyprus par Socrates Evangelides, intitulé « Sour Grapes not Bitter Lemons ». Voir David Roessel, « Rodis Roufos on Bitter Lemons: A Suppressed Section of The Age of Bronze ", in Deus Loci: The Lawrence Durrell Journal, n³ 3, 1994a, p. 130.

2- David Roessel (éd.), « Letters of Lawrence Durrell to Austen Harrison », in Deus Loci..., op. cit., 1994b, p. 22-23, cité par Fotini

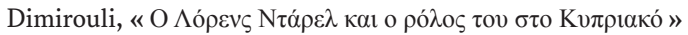
(Lawrence Durrell et son rôle dans l'affaire chypriote), in Journal Avgi, 16/09/2012.

3- Lawrence Durrell, Citrons acides, Paris, Buchet/Chastel, 1995 [1 ${ }^{\text {ère }}$ éd. 1975], p. 18

4- Durrell, op. cit., p. 26

5- Camille Enlart, L'art gothique et la Renaissance en Chypre, Paris, Ernest Leroux, 1899

6- Cf. David Roessel, «'This is not a political book': Bitter Lemons as British propaganda ", in Byzantine and Modern Greek Studies, n ${ }^{\circ} 24$, 2000, p. 235-245

7- Alexandre-Garner cite la lettre officielle de candidature de Durrell au poste de gouverneur de Chypre, « dans laquelle il explique pourquoi il est la personne idéale pour le poste " ; Corinne Alexandre-Garner, Lawrence Durrell. Dans l'ombre du soleil grec, Paris, La Quinzaine littéraire/L. Vuitton, 2012, p. 204-205.

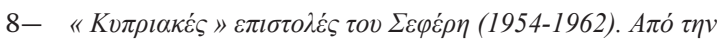

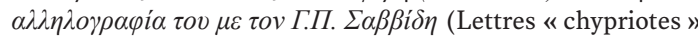
de Séféris (1954-1962). Extraits de sa correspondance avec G. P. Savvidis), édité par Katerina Kostiou, Nicosie, Politistiko Idryma Trapezis Kyprou, 1991, p. 57-58, cité par Dimirouli, op. cit.

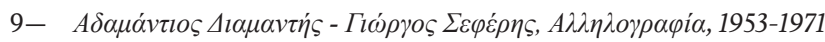
(Adamantios Diamantis - Georges Séféris, Correspondance, 19531971), édité par Michalis Pieris, Athènes, Stigmi, 1985, p. 35, cité par Dimirouli, op. cit.

10- Durrell, op. cit., p. 187-188

11- Durrell, op. cit., p. 283

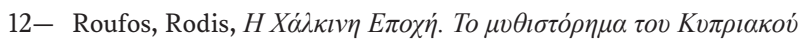
$A \gamma \omega ́ v \alpha$ (L'Âge de bronze. Le roman de la luttre chypriote), Athènes, Estia, 1960

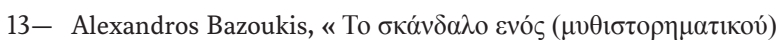

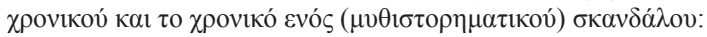

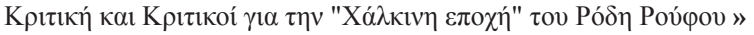
[Le scandale d'une chronique (romanesque) et la chronique d'un scandale (romanesque) : critique et critiques sur L'Âge de bronze de Rodis Roufos], in Nea Estia, n ${ }^{\circ}$ 1856, décembre 2012, p. 142-201

14- Roessel, 1994a, op. cit., p. 132-133

15- Roessel, 1994a, op. cit., p. 129-130. Roessel se demande d'ailleurs « ce qui était tellement offensif dans ces pages en 1960 » (ibid., p. 130).

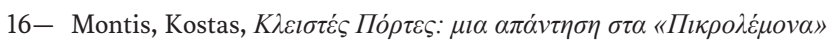

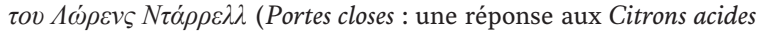
de Lawrence Durrell), Nicosie, 1964. Traduit en anglais par David Roessel et Soterios G. Stavrou, Closed Doors. An Answer to Bitter Lemons by Lawrence Durrell, Minneapolis, Nostos Books, 2004. 